\title{
SNAKE ROBOT LOCOMOTION PATTERNS FOR STRAIGHT AND CURVED PIPE
}

\author{
VIRGALA Ivan ${ }^{1}$, LIPTÁK Tomáš ${ }^{1}$, MIKOVÁ Lubica ${ }^{1}$ \\ ${ }^{1}$ Technical University of Košice, Faculty of Mechanical Engineering, Department of Mechatronics, Park \\ Komenského 8,04200 Košice, Slovak Republic, email: ivan.virgala@tuke.sk
}

\begin{abstract}
In the paper the locomotion of snake robot is introduced considering locomotion in straight and curved pipe. For the straight pipe locomotion was designed traveling wave locomotion pattern with sine-like wave which expands from rear of the robot to its front. For the locomotion in curved pipe was designed approach which is based on inverse kinematic model including besides primary task also secondary tasks, namely kinematic singularities avoidance task, obstacle avoidance task and joint limit avoidance task. For final inverse kinematic model was used approach of weight matrices by which can be stated the priorities of particular tasks. Both case studies were tested by experimental snake robot in order to verify introduced methodology for locomotion in the straight and curved pipe.
\end{abstract}

KEYWORDS: inverse kinematic model, path planning, potential field method, snake robot, weight matrix

\section{Introduction}

A few decades ago the researches started designing of service robots, those mechanics of motion has been inspired by nature. One kind of these robots are snake robots which imitate body of the biological snake. The snake robots usually consist of many same links. Although the rotation of two adjoining joints are small, by considering many of links can snake robot create the shape of required curve. The kinematic structure of snake robots are suitable for hardto-reach areas like channels or pipes. Its kinematic structure is also suitable for medical robots during surgical operation as well as in military area. The snake robots have potential to overcome roughness of terrain in which they are applied. Considering a high number of degrees of freedom can snake robots be more flexible and adaptable to their environment. The example is a searching of survivor in the case of building crashing.

Prof. Shiego Hirose is considered to be the first designer of snake robot. His first robot with the name ACM III was designed in 70s [1]. This robot used passive wheels which imitate the obstacles by which can snake robot generate forward motion. From this moment there were designed a plenty of snake robots which use passive or active wheels. Besides them were developed snake robots without any wheels which use contact with their environment for forward motion.

The aim of this study is to investigate locomotion of snake robot in the pipe. From this reason there will be described some works dealing with our research area. In the paper [2] the authors dealt with micro snake robot which was able to pass through the pipe. The pipe changes its diameter and the snake robot has to overcome these changes. Designed snake robot within this work performed motion by generating of sinusoidal curve. In [3] the authors investigated mapping task of the snake robot moving in confined area. In following work [4] was designed snake robot for experimental purposes. The authors investigated motion of robot in inclined pipe with different diameters. Within this research were experimentally tested several curves of 
snake robot backbone during its forward locomotion. For the pipe inspection was also designed snake robot using active wheels in [5].

Our research team has also contributed to this area. In [6] we have designed snake robot LocoSnake having one revolute as well as one prismatic joint in each robot link. This solution was unique in the time of LocoSnake development. The robot was designed for experimental purposes moving in the pipe with rectangular cross-section. In mentioned research was experimentally tested concertina locomotion using revolute and prismatic joints. By LocoSnake were also tested different curves of snake robot backbone due to fixation of the robot in the pipe during its locomotion [7]. For measuring of fixation quality the digital image correlation method was used. Within this research was investigated only motion in the straight pipe. Considering above mentioned research works as well as our own contribution in this area, we have designed new snake robot for experimental purposes. The main requirement on the new designed robot is motion in the straight and curved pipe with rectangular cross-section. Within this research we have derived mathematical model for snake robot locomotion, which was experimentally verify by new designed snake robot.

In the paper, at first locomotion patterns for straight and curved pipe is introduced. Next, path planning task of snake robot links is introduced using potential field method. The mathematical model for locomotion in curved pipe is derived. Derived inverse kinematic model is verified by experimental model of snake robot passing through the straight and curved pipe.

\section{Locomotion in straight and curved pipe}

The paper investigates locomotion of snake robot through the straight pipe as well as curved pipe. The locomotion in the straight pipe is not too difficult from the view of control system. Since our developed snake robot does not have passive wheels, we have to use its environment for forward motion. As have been mentioned, passive wheels play role in the replacing of the contact between the snake and obstacle. The snake robot without passive wheels uses obstacle in its environment in order to produce propulsive force. Considering the locomotion in the pipe the snake robot uses contact between particular robot links and walls of the pipe. Biologically snake moving in the pipe uses locomotion pattern, so-called concertina locomotion [1][8]. Assuming joint range of our experimental snake robot and diameter of the pipe, the concertina locomotion would be not very effective. From this reason we have used locomotion pattern which has not basic in the nature like concertina locomotion. For our analysis the traveling wave locomotion pattern is used. This locomotion pattern considers sine-like wave moving from the rear of the robot to its front, see Fig. 1.

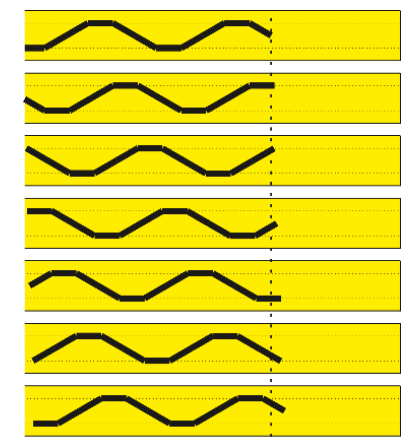

Fig. 1 Traveling wave locomotion pattern

The motion introduced in the Fig. 1 is based on friction forces between the snake robot links and walls of the pipe. The sine-like wave expands from the rear to front of the robot. As can be seen from the Fig. 1, at every moment of locomotion there are several points on the snake robot 
which are in the contact with vertical part of the pipe. For this reason can snake robot generates propulsive force.

More complicated is situation when the robot has to pass through the curved pipe. Now it is important to design a locomotion pattern for passing this elbow, what is not so trivial. There are many factors which influences this locomotion situation. Let's consider that robot passes through straight pipe. At the end of straight part of the pipe is elbow. Snake robot always stops in different distance between end of the pipe, and it has always different kinematic configuration. From this reason it is important to design locomotion pattern which would deals with mentioned aspects. In other words, we can say that forward locomotion in the straight pipe can be performed by programming of control system which assumes only diameter of the pipe and diameter of the snake robot link. By cyclic performing of programmed algorithm will robot moves forward. Different situation is in the case of pipe elbow. Considering above mentioned aspects of this case we have designed mathematical model assuming inverse kinematics. By inverse kinematic model can control system deals with different situation of snake robot initial position before passing the pipe elbow. Designed locomotion pattern for pipe elbow can be seen in the Fig. 2.

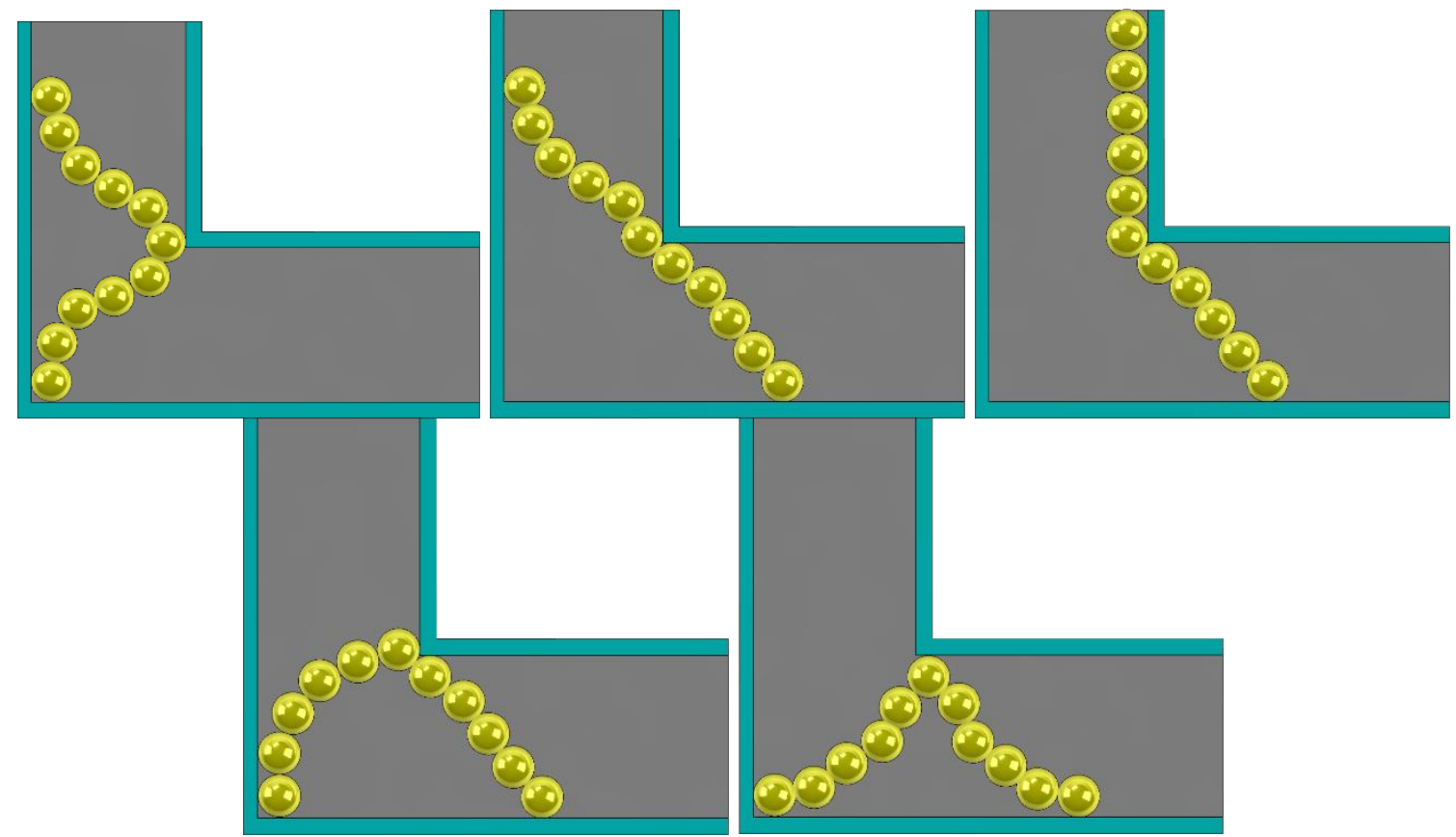

Fig. 2 Locomotion pattern for passing the curved pipe

Let's consider situation described in the Fig. 2. In the position 1 is snake robot at the end of the pipe. Snake robot tries to take position such that by links 1-6 could be robot fixed in the pipe. In the position 2, while rear of the robot is fixed, the links 7-11 move to curved part. In the position 3 is robot fixed in the pipe by 7-11 links and links 1-6 move parallel with the axis of the pipe. The position 4 shows moving of the $1^{\text {st }}$ link to the corner of the pipe. In the position 5 has robot initial position for forward motion. To attainment described locomotion pattern we used inverse kinematic model. In particular positions described in the Fig. 2 has snake robot fixed links as well as moving links. For example for position $1: 6^{\text {th }}$ link represents base and $11^{\text {th }}$ link represents end-effector. The aim is to move $11^{\text {th }}$ link into contact with wall of the pipe in order to ensure fixation of 7-11 links. Then can move links 1-6 according to position 2. Now the task is to perform mentioned links motions and avoid any collisions with the pipe and also ensures joints range of motion. For this reason we have designed inverse kinematic model dealing with all described requirements. Before the inverse kinematic model will be introduced there has to be determined path for links for particular positions of the Fig. 2. 


\section{Path planning for passing of the pipe elbow}

The inverse kinematic model for passing the pipe elbow will use the path, planned for particular positions of the designed locomotion pattern (Fig. 2). Let's consider the position 12 , the aim of this section is to determine the path of $11^{\text {th }}$ link from initial position (position 1) to final position (position 2). This idea is used for every positions from the Fig. 2. To the path planning task we have used potential field method.

\subsection{Potential field method}

The basic idea of potential field method arise from the nature. As an example we can mention magnetic field or ball rolling in a hill. This approach is often used in robotics. The main idea is based on attractive and repulsive potential field. Attractive field is a function of goal position of the robot. Whole workspace of the robot is divided by grid with defined separation [14]. To design of the attractive field is necessary to express relation between each point of the grid in relative to the goal position of the robot. The function representing attractive field has its minimum in goal position, see Fig. 6 and Fig. 7. Along with attractive field there also repulsive field [13]. Repulsive field includes all obstacles in robot workspace. In our study the obstacles are represented by walls of the pipe. In the center of particular obstacles the repulsive force affects out of these obstacles.

The attractive field can be expressed as [9]

$$
U_{a t t}(\mathbf{s})=\frac{1}{2} \xi\left\|\mathbf{s}_{\text {goal }}-\mathbf{s}_{\text {grid }}\right\|^{k}
$$

where $\mathbf{s}_{\text {goal }}$ and $\mathbf{s}_{\text {grid }}$ are goal position of the robot and each position of grid relative to the goal position, respectively. By scalar variable $k$ can be change the shape of the attractive field. $\xi$ is positive scalar factor. Based on equation (1) can be derived corresponding attractive force what is a negative gradient of attractive field [10]

$$
\mathbf{F}_{a t t}(\mathbf{s})=-\nabla U_{a t t}(\mathbf{s})=-\frac{\partial U_{a t t}(\mathbf{s})}{\partial \mathbf{s}}
$$

Repulsive field can be expressed as

$$
U_{\text {rep }}(\mathbf{s})=\left\{\begin{array}{c}
\eta\left(\frac{1}{d}-\frac{1}{d_{0}}\right)^{2}, d \leq d_{0} \\
0, d>d_{0}
\end{array}\right.
$$

where $d$ is distance between robot and obstacle what is expressed as $d=\left\|\mathbf{s}_{\text {obstacle }}-\mathbf{s}_{\text {robot }}\right\|$. By parameter $d_{0}$ is expressed influence of the obstacle. $\eta$ is scalar parameter. Repulsive force is negative potential of repulsive field

$$
\nabla U_{r e p}(\mathbf{s})=\left\{\begin{array}{c}
\eta\left(\frac{1}{d}-\frac{1}{d_{0}}\right)^{2} \frac{\left(\mathbf{s}-\mathbf{s}_{0}\right)}{d^{3}}, d \leq d_{0} \\
0, d>d_{0}
\end{array}\right.
$$

where $\mathbf{s}_{0}$ is distance to the obstacle and $\mathbf{s}$ is position of the robot. Final potential field is given by sum of attractive and repulsive field

$$
U_{\text {total }}(\mathbf{s})=U_{a t t}(\mathbf{s})+U_{\text {rep }}(\mathbf{s})
$$

The output of the path planning task is matrix $\mathbf{P} \in \mathbb{R}^{p \times m}$, where $p$ is number of points between starting and goal position of the robot and $m$ is dimension of performed task what is in the case of planar task $m=2$. 
The example of the repulsive field which shapes the elbow of the pipe can be seen in the Fig. 3 and Fig. 4.

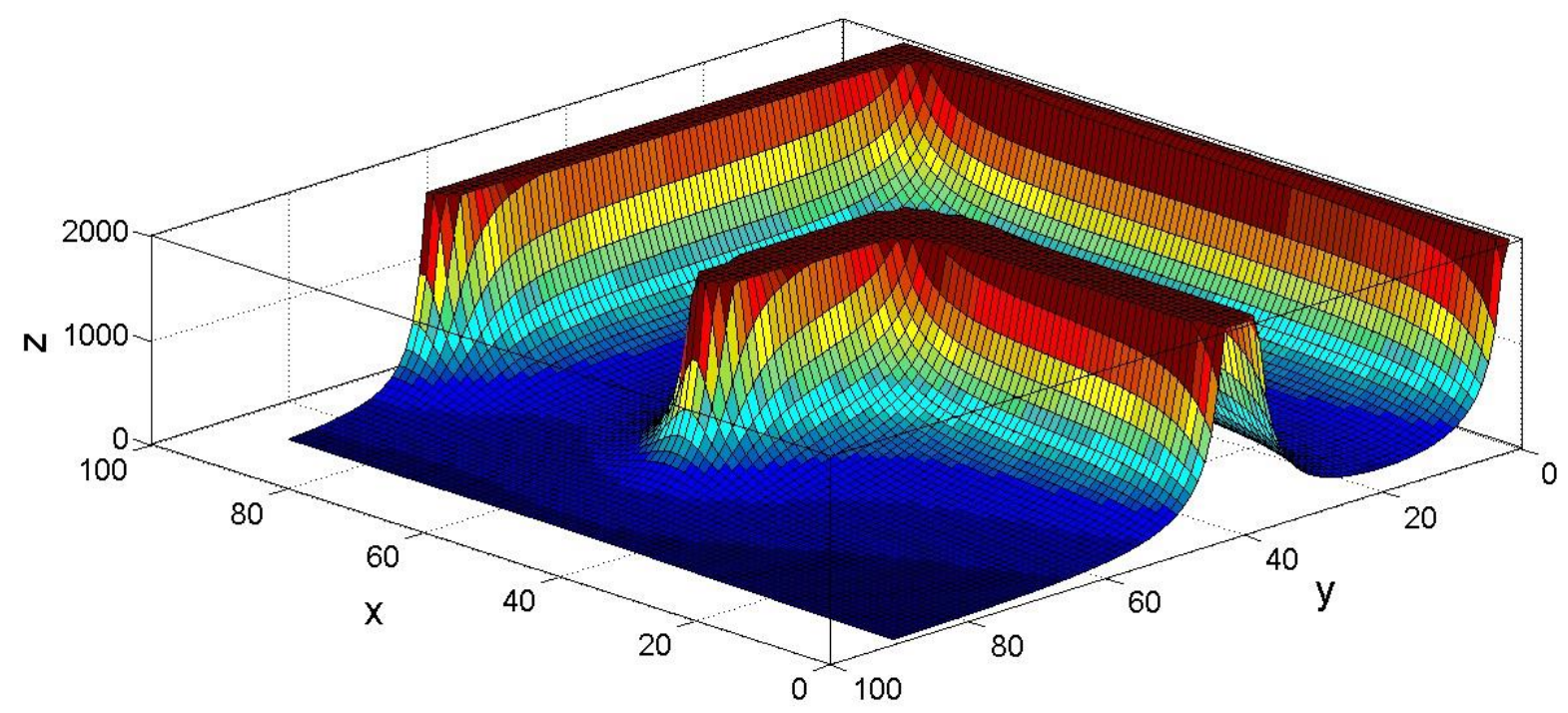

Fig. 3 Pipe modeled by repulsive forces

Model of the pipe is expressed by matrix $\mathbf{O} \in \mathbb{R}^{h \times m}$, where $\mathrm{h}$ is the number of circles of which the pipe is modeled and $m$ is dimension of the task $(m=2)$.

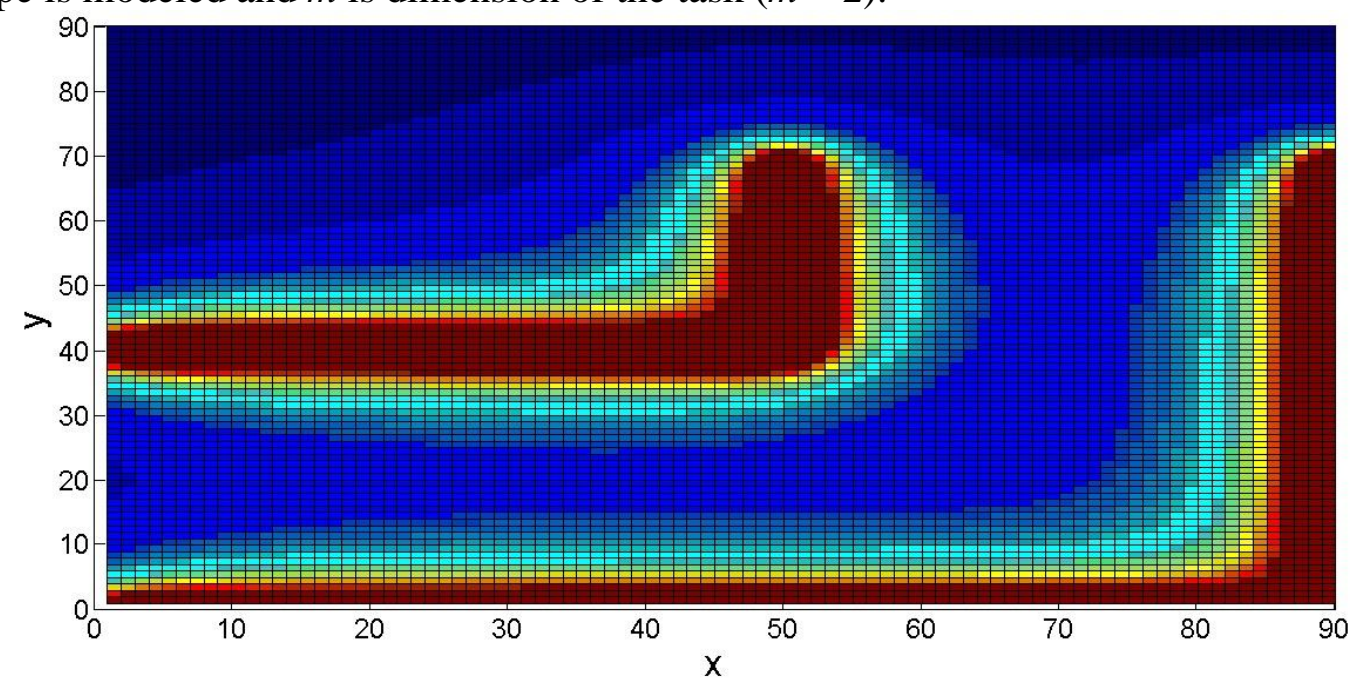

Fig. 4 Pipe modeled by repulsive forces - top view

The pipe is modeled by following way. The walls of the pipe are replaced by circles with specified diameter. Distance between centers of adjacent circles is diameter of the circle. The example of attractive field in the Fig. 5 and Fig. 6 is shown. 


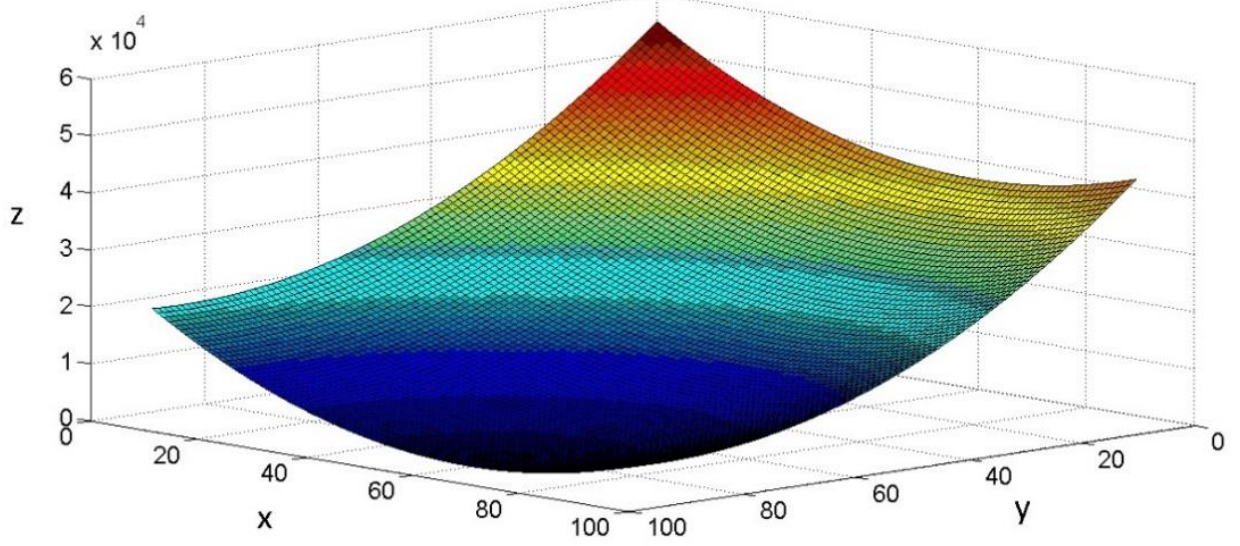

Fig. 5 Example of attractive field

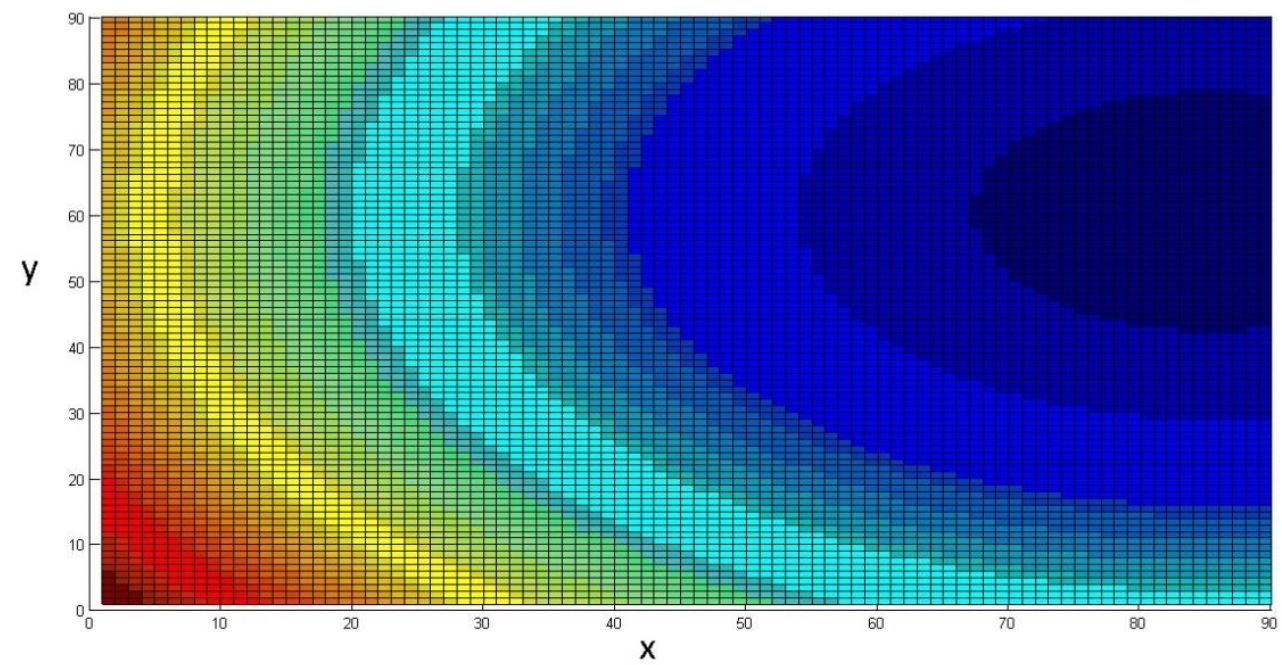

Fig. 6 Example of attractive field - top view

The goal position of the robot is specified by dark blue color in the Fig. 5 and Fig. 6. The example of final potential field of particular situation from the Fig. 2 in the Fig.7 is shown.

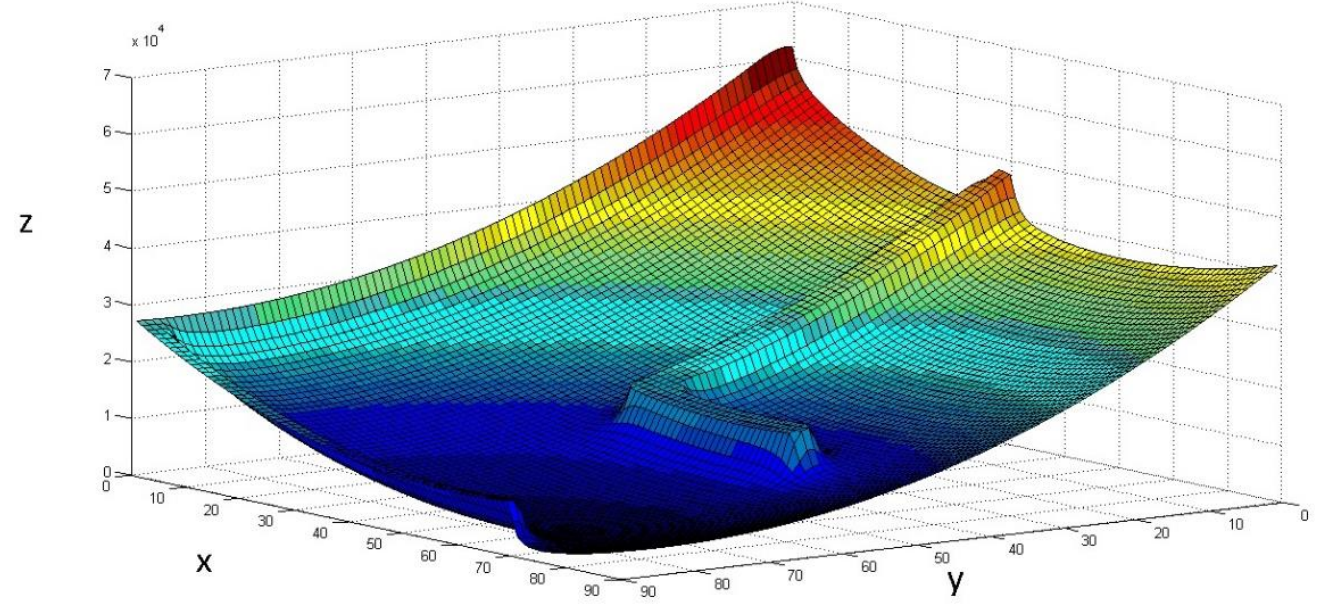

Fig. 7 Example of final potential field

Considering positions 1-2 from the Fig. 2, the potential field in software Matlab is modeled, see Fig. 8. 


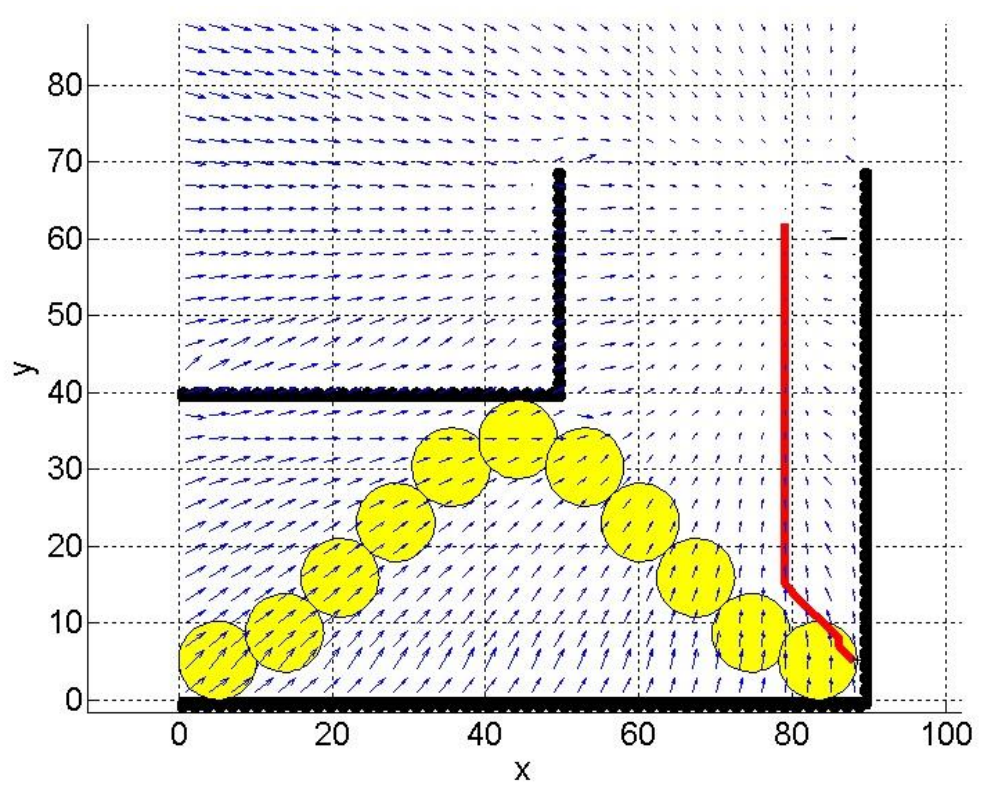

Fig. 8 Example of final potential field for snake robot passing the pipe elbow

The red color in the Fig. 8 represents planned path of the $11^{\text {th }}$ link during position 2 (Fig. 2). Every situation from the Fig. 2 has to deal with path planning described in this section. This section describes only motion from position 1 to position 2. Path planning for other positions are derived by the same way. The main contribution of path planning is matrix $\mathbf{P} \in \mathbb{R}^{p x m}$ what is the input to the inverse kinematic model.

\section{$4 \quad$ Inverse kinematic model with consideration of secondary tasks}

In the previous sections has been introduced the concept of this research, what is snake robot locomotion in the straight as well as curved pipe with rectangular cross-section. For the locomotion through the straight pipe is necessary to know basic parameters like diameter of the pipe and length of snake robot link. Based on these constants can be programmed controller for locomotion, which doesn't have to deal with other conditions of robot environment. For passing through the curved pipe we have designed new approach which is based on inverse kinematic model. Derived inverse kinematic model can be divided into two tasks. The first task, namely primary task, deals with finding such generalized variables vector $\mathbf{q} \in \mathbb{R}^{n}$ ( $n$ - number of DOF) by which specific robot link reaches desired position in the pipe. Inverse kinematic model is based on following equation

$$
\Delta \mathbf{x}=\mathbf{J}(\mathbf{q}) \Delta \mathbf{q}
$$

where $\mathbf{q} \in \mathbb{R}^{n}$ and $\mathbf{x} \in \mathbb{R}^{m}$.

Next task namely secondary task can includes several tasks what in our case are kinematic singularity avoidance task, joint limit avoidance task and obstacle avoidance task. These tasks will be described in the following sections.

\subsection{Kinematic singularity avoidance task}

Kinematic singularity means difficulties with mapping of Cartesian space in to joint space. At singular configuration of robot the mobility is significantly reduced. A singularity occurs when joint velocity in joint space becomes infinite to maintain Cartesian velocity. This situations arise whenever the determinant of Jacobian matrix $\mathbf{J}(\mathbf{q})$ equals zero. To solve presented problem we can use damped least squares method, which was first time used for inverse kinematics by Wampler [11] and Nakamura and Hanfusa [12]. The objective function is stated by equation (6) 


$$
F=\|\mathbf{J} \dot{\mathbf{q}}-\dot{\mathbf{x}}\|^{2}+\|\alpha \dot{\mathbf{q}}\|^{2}
$$

where $\|$.$\| denotes Euclidean norm. By solving equation (7) according to \frac{d F}{d \dot{\mathbf{q}}^{T}}=0$ we can get solution by which can be dealt with kinematic singularities

$$
\dot{\mathbf{q}}=\mathbf{J}^{T}\left(\mathbf{J J}^{T}+\alpha^{2} \mathbf{I}\right)^{-1} \dot{\mathbf{x}}
$$

where $\alpha$ is damping constant.

\subsection{Obstacle avoidance task}

Considering the robot locomotion through the curved pipe, of course, there will be not considered obstacles in the pipe. As obstacles will be considered walls of the pipe. In previous section has been mentioned snake robot uses walls of the pipe for generating propulsive force for forward locomotion. On the other hand, during passing the curved part of the pipe, the walls of the pipe play role as obstacles for moving parts of the snake robot while static parts of the snake robot are fixed between walls of the pipe. In the following section model of obstacle avoidance task is introduced. Algorithm for finding potential point of collision can be described as follows.

If we consider i-th link which is between i-th joint with coordinates $\mathbf{s}_{i}$ and (i+1)-th joint with coordinates $\mathbf{s}_{i+1}$, then can be expressed unit vector $\mathbf{e}_{i}$ which determines the direction of $i$-th robot link

$$
\mathbf{e}_{i}=\frac{\mathbf{s}_{i+1}-\mathbf{s}_{i}}{L_{i}}
$$

where $L_{i}$ is length of snake robot link.

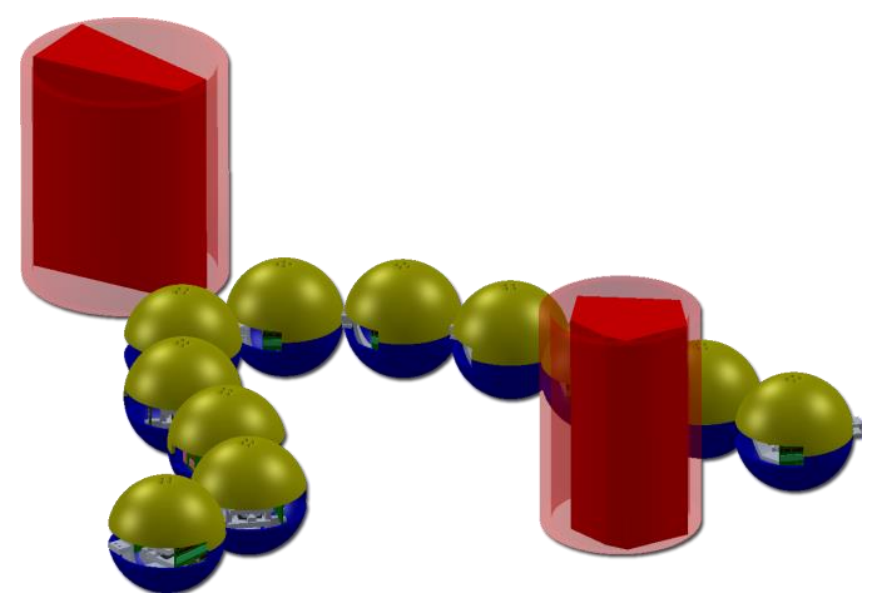

Fig. 9 Snake robot with obstacles

Next point is to characterize an obstacle. In general, let's obstacle has an irregular shape. To simplify the description of an obstacle we can assume cylinder around an irregular shape of all obstacles. This simplification is the same as we have noticed in previous section when we have designed the walls of the pipe by repulsive field. Now, as the center of obstacle we can consider the center of the cylinder, which has coordinate $\mathbf{s}_{o}$. The projection of line from i-th joint of the snake robot to center of particular cylinder on the $\mathrm{i}$-th link is

$$
p_{i}=\mathbf{e}_{i}^{T}\left(\mathbf{s}_{0}-\mathbf{s}_{i}\right)
$$


Now, coordinate of potential point of snake robot which can reach collision with the obstacle is expressed as

$$
\mathbf{s}_{c i}=\mathbf{s}_{i}+p_{i} \mathbf{e}_{i}
$$

The distance between potential point of collision on the snake robot and center of cylinder (obstacle) is

$$
d_{c i}=\left\|\mathbf{s}_{c i}-\mathbf{s}_{0}\right\|
$$

The unit vector of potential point of collision to center of the obstacle is

$$
\mathbf{u}_{i}=\frac{\mathbf{s}_{a i}-\mathbf{s}_{0}}{d_{c i}}
$$

To complete the mathematical model of obstacle avoidance task is necessary to determine particular Jacobian matrix. For i-th row of Jacobian matrix can be written

$$
\mathbf{J}_{c i}=-\mathbf{u}_{i}^{T} \mathbf{J}_{\mathbf{s}_{c i}}
$$

where

$$
\mathbf{J}_{\mathbf{s}_{c i}}=\frac{\partial \mathbf{s}_{c i}}{\partial \mathbf{q}}
$$

Jacobian matrix $\mathbf{J}_{c}$ will be now consists of submatrices $\mathbf{J}_{c i} \cdot \mathbf{J}_{c} \in \mathbb{R}^{l \times l}$ where $l$ represents number of snake robot links, which could get in collision with the obstacles.

\subsection{Joint limit avoidance task}

The aim of this secondary task is to prevent to snake robot joint to reach angular rotation which is out of the range of the joint. Experimental snake robot used for our investigation has limitation of its rotation $\pm 45^{\circ}$. The effort of this secondary task is to hold snake robot joints in this range.

To solve this problem will be used weight variable. We can divide whole joint range into 4 positions, namely admissible rotation, rotation on boundary and rotation out of the joint range. While joint is in the admissible rotation, the weight variable equals zero. When joint reaches rotation on boundary of the range, the weight variable has higher value in comparison with the first case. When the joint is out of the range the weight variable has maximum value. Mentioned approach can be expressed by equation (16)

$$
\mathrm{W}_{l i}=\left\{\begin{array}{c}
W_{w} \leftarrow q_{i}<q_{\text {imin }} \\
\frac{W_{w}}{2}\left\{1+\cos \left[\pi\left(\frac{q_{i}-q_{\text {imin }}}{\rho_{i}}\right)\right]\right\} \leftarrow q_{\text {imin }} \leq q_{i} \leq q_{\text {imin }}+\rho_{i} \\
0 \leftarrow q_{\text {imin }}+\rho_{i}<q_{i}<q_{\text {imax }}-\rho_{i} \\
\frac{W_{w}}{2}\left\{1+\cos \left[\pi\left(\frac{q_{\text {imax }}-q_{i}}{\rho_{i}}\right)\right]\right\} \leftarrow q_{\text {imax }}-\rho_{i} \leq q_{i} \leq q_{\text {imax }} \\
W_{w} \leftarrow q_{i}>q_{\text {imax }}
\end{array}\right.
$$

Weight matrix $\mathbf{W}_{l}$ is diagonal matrix consisting of particular weight variables $\mathbf{W}_{l i}$

$$
\mathbf{W}_{l}=\left[\begin{array}{lllll}
\mathrm{W}_{l 1} & & & & \\
& \mathrm{~W}_{l 2} & & & \\
& & \mathrm{~W}_{l 3} & & \\
& & & \cdots & \\
& & & & \mathrm{W}_{l n}
\end{array}\right]
$$

Weight matrix $\mathbf{W}_{l} \in \mathbb{R}^{n \times n}$ is used with cooperation Jacobian matrix $\mathbf{J}_{l} \in \mathbb{R}^{n \times n}$. Jacobian matrix for this task is $\mathbf{J}_{l}=\frac{\partial \mathbf{e}}{\partial \mathbf{q}}$, where $\mathbf{e}$ characterizes if particular joint considers the joint limit 
avoidance task. If particular joint doesn't consider the joint limit avoidance task, its component in Jacobian matrix $\mathbf{J}_{l}$ is zero, otherwise it is one.

\subsection{Final inverse kinematic model}

The final inverse kinematic model consists of primary and secondary tasks. All above investigated tasks will be now used in final model. The final inverse kinematic model can arise from the same idea as have been described in the section 4.1. The objective function dealing with primary as well as all secondary tasks is expressed as

$$
F=\|\mathbf{J} \dot{\mathbf{q}}-\dot{\mathbf{x}}\|^{2}+\|\alpha \dot{\mathbf{q}}\|^{2}+\left\|\mathbf{J}_{c} \dot{\mathbf{q}}-\dot{x}_{c}\right\|^{2}+\left\|\mathbf{J}_{l} \dot{\mathbf{q}}-\dot{x}_{l}\right\|^{2}
$$

Equation (18) can be expanded into following form

$$
\begin{gathered}
F=(\mathbf{J} \dot{\mathbf{q}}-\dot{\mathbf{x}})^{T} \mathbf{W}_{e}(\mathbf{J} \dot{\mathbf{q}}-\dot{\mathbf{x}})+(\dot{\mathbf{q}})^{T} \mathbf{W}_{s}(\dot{\mathbf{q}})+\left(\mathrm{J}_{c} \dot{\mathbf{q}}-\dot{x}_{c}\right)^{T} \mathbf{W}_{c}\left(\mathrm{~J}_{c} \dot{\mathbf{q}}-\dot{x}_{c}\right) \\
+\left(\mathbf{J}_{l} \dot{\mathbf{q}}-\dot{x}_{l}\right)^{T} \mathbf{W}_{l}\left(\mathbf{J}_{l} \dot{\mathbf{q}}-\dot{x}_{l}\right)
\end{gathered}
$$

where matrices $\mathbf{W}_{e} \in \mathbb{R}^{m \times m}, \mathbf{W}_{s} \in \mathbb{R}^{n \times n}, \mathbf{W}_{c} \in \mathbb{R}^{n \times n}, \mathbf{W}_{l} \in \mathbb{R}^{n \times n}$ are weight matrices for primary and particular secondary tasks. By this approach can be stated priority of each task. The objective function $F$ is minimized by derivation of $F$ which equals zero.

$$
\frac{d F}{d \dot{\mathbf{q}}^{T}}=2\left(\mathbf{J}^{T} \mathbf{W}_{e} \mathbf{J}+\mathbf{J}_{c}^{T} \mathbf{W}_{c} \boldsymbol{J}_{c}+\mathbf{J}_{l}^{T} \mathbf{W}_{l} \boldsymbol{J}_{l}+\mathbf{W}_{s}\right) \dot{\mathbf{q}}-2\left(\mathbf{J}^{T} \mathbf{W}_{e} \dot{\mathbf{x}}+\mathbf{J}_{c}^{T} \mathbf{W}_{c} \dot{x}_{c}+\mathbf{J}_{l}^{T} \mathbf{W}_{l} \dot{x}_{l}\right)
$$

From the equation (20) the joints velocities are

$$
\dot{\mathbf{q}}=\left(\mathbf{J}^{T} \mathbf{W}_{e} \mathbf{J}+\mathbf{J}_{c}^{T} \mathbf{W}_{c} \boldsymbol{J}_{c}+\mathbf{J}_{l}^{T} \mathbf{W}_{l} \boldsymbol{J}_{l}+\mathbf{W}_{s}\right)^{-1}\left(\mathbf{J}^{T} \mathbf{W}_{e} \dot{\mathbf{x}}+\mathbf{J}_{c}^{T} \mathbf{W}_{c} \dot{x}_{c}+\mathbf{J}_{l}^{T} \mathbf{W}_{l} \dot{x}_{l}\right)
$$

Since, joint velocities have to be vanished when joint limit or obstacles occurs, the velocities for secondary tasks $\dot{x}_{c}$ and $\dot{x}_{l}$ have to be selected to be zero. Based on this idea can be written final inverse kinematic model for our case study

$$
\dot{\mathbf{q}}=\left(\mathbf{J}^{T} \mathbf{W}_{e} \mathbf{J}+\mathbf{J}_{c}^{T} \mathbf{W}_{c} \boldsymbol{J}_{c}+\mathbf{J}_{l}^{T} \mathbf{W}_{l} \boldsymbol{J}_{l}+\mathbf{W}_{s}\right)^{-1}\left(\mathbf{J}^{T} \mathbf{W}_{e} \dot{\mathbf{x}}\right)
$$

Final inverse kinematic model according to equation (22) will be now used for experimental verification in the following section.

\section{$5 \quad$ Experimental verification of inverse kinematic model}

Our methodology can be now verified by experimental analysis. For experiments was used snake robot developed in Department of Mechatronics in Faculty of Mechanical Engineering (Technical University of Košice). Developed snake robot consists of 11 same links which are controlled by microcontrollers ATmega8-16PU at frequency $16 \mathrm{MHz}$. The links have spherical 
cover with diameter $102 \mathrm{~mm}$. Spherical cover is created from material ABS by rapid prototyping technology. The mathematical model derived in previous section runs in software Matlab. The computer with Matlab communicates with snake robot by Universal Synchronous / Asynchronous Receiver and Transmitter (USART) interface. For experiments was designed modular pipe built from profiles of height $80 \mathrm{~mm}$.

For testing of locomotion in the straight pipe was built the pipe with diameter of $170 \mathrm{~mm}$ with rectangular cross-section. Based on diameter of the pipe and diameter of robot link was modify model of traveling wave. The sine-like wave expands from rear of the snake robot to its front link while particular links are always in the contact with walls of the pipe. The result of straight pipe locomotion in the Fig. 11 is shown.

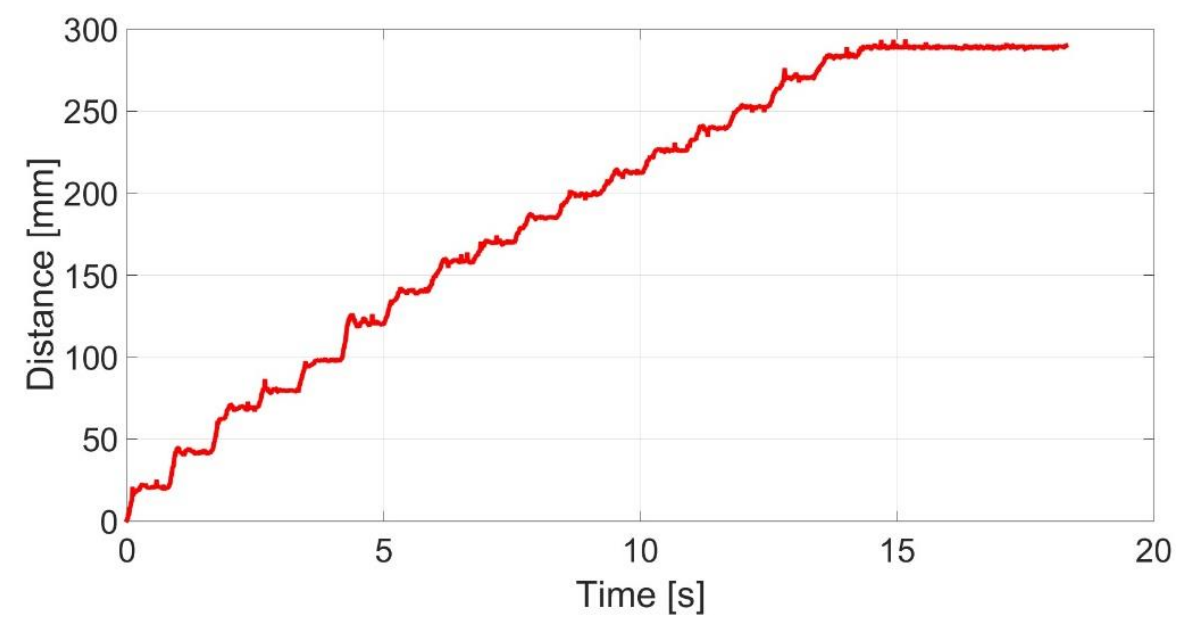

Fig. 11 Travelled distance in the straight pipe - example of locomotion

The shots from the straight pipe locomotion in the Fig. 12 are shown.

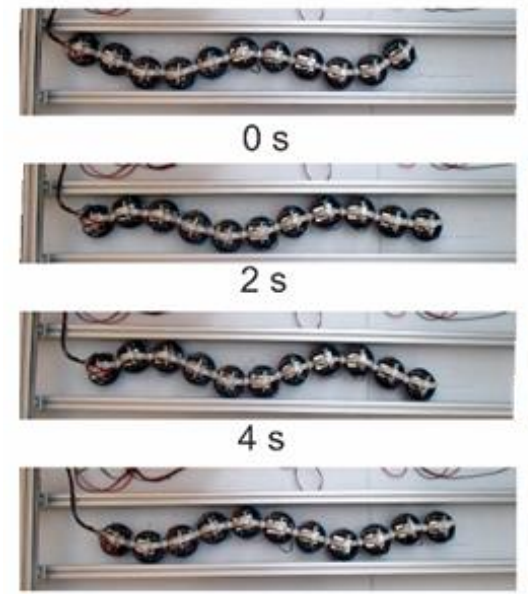

$6 s$

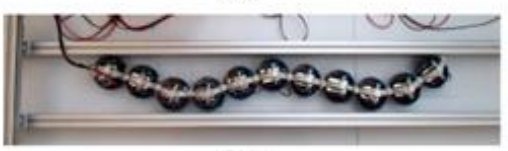

$8 \mathrm{~s}$
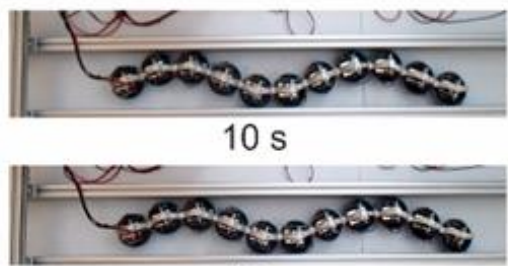

$12 \mathrm{~s}$

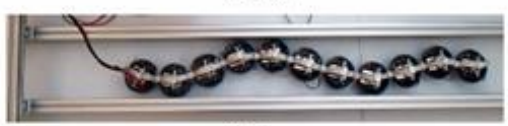

$14 \mathrm{~s}$

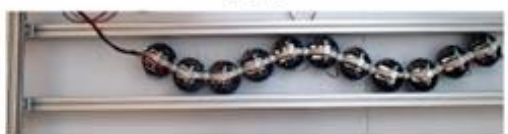

$16 \mathrm{~s}$

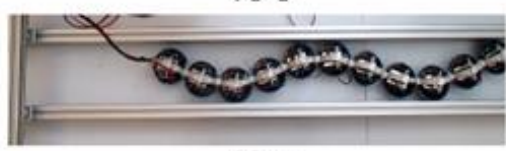

$18 \mathrm{~s}$
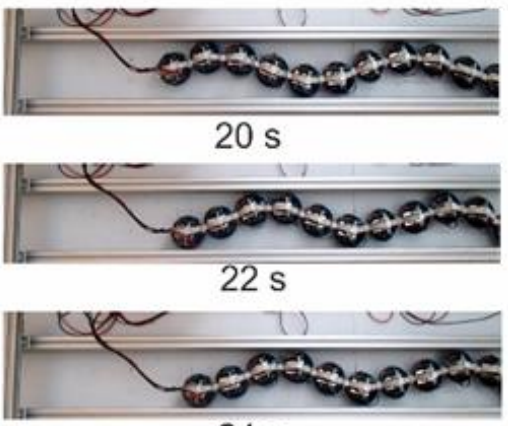

$24 \mathrm{~s}$

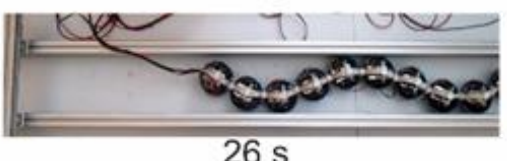

$26 s$

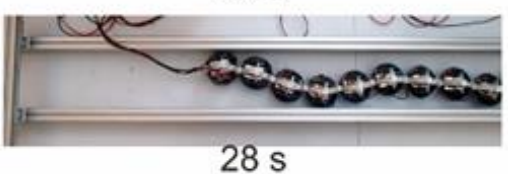

Fig. 12 Locomotion of snake robot in the straight pipe

For the passing of the pipe elbow was built next modular pipe. The diameter of the pipe was $390 \mathrm{~mm}$. Within this case study the control system used mathematical model described by equation (22). The shots from the second case study in the Fig. 13 are shown. 


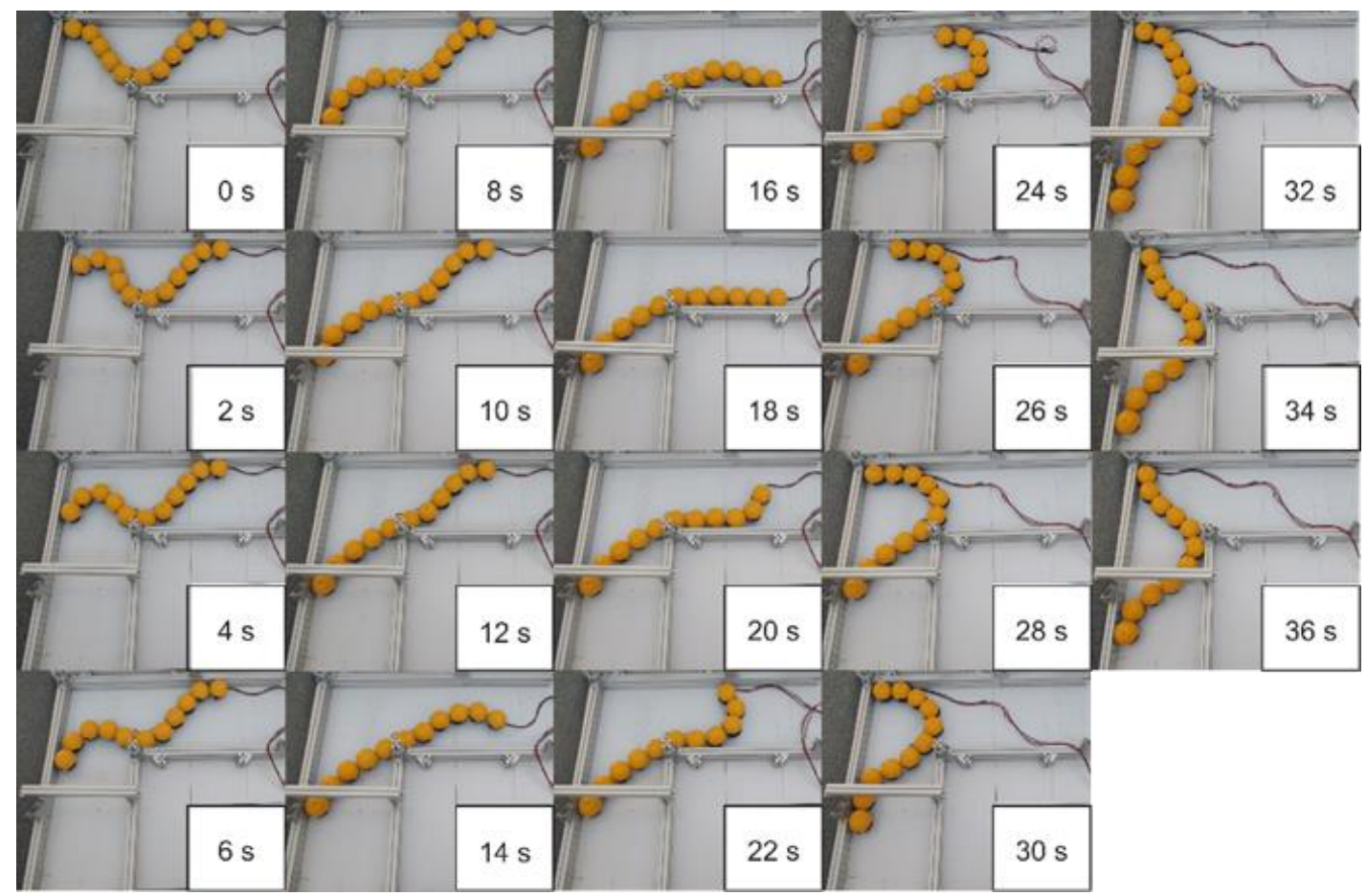

Fig. 13 Passing through the pipe elbow

The Fig. 13 corresponds with the description of the Fig. 2 where was designed the locomotion pattern for passing the pipe elbow.

\section{CONCLUSION}

The paper presents locomotion of the snake robot in the straight and also curved pipe. For straight pipe locomotion we used traveling wave of sine-like wave which expands from rear link up to front link of snake robot. By contacts between robot links and walls of the pipe is generated propulsive force for forward locomotion. We have designed methodology for passing the pipe elbow which is based on inverse kinematic model. Final inverse kinematic model deals with primary task as well as secondary tasks, what are namely kinematic singularity avoidance task, obstacle avoidance task and joint limit avoidance task. The input to inverse kinematic model is output from the task of path planning. We have modeled the path by potential field method. Inverse kinematic model was based on minimizing of objective function which consists of all mentioned tasks. To the derived solution were added weight matrices by which can be determined the priority of particular tasks.

Introduced locomotion patterns for straight and curved pipes were tested by experimental snake robot developed in Department of Mechatronics / Faculty of Mechanical Engineering (Technical University of Košice). The aim of the experiments was not to precise path tracking but testing of designed methodology. Both case studies was verified by means of modular pipes and the results show that methodology is suitable. In the future work our snake robot should moves in the pipe with higher degree of autonomy based on camera and sensors.

\section{Acknowledgement}

This paper was supported by project VEGA1/0872/16 "Research of synthetic and biological inspired locomotion of mechatronic systems in rugged terrain" and project VEGA 1/0389/18 "Research on kinematically redundant mechanisms". 


\section{REFERENCES}

[1] S. Hirose. Biologically Inspired Robots: Snake-like Locomotors and Manipulators, Oxford University Press, Oxford, 1993.

[2] S. Wakimoto, J. Nakajima, M. Tanaka, T. Kanda, K. Suzumori. A micro snake-like robot for small pipe inspection, MHS 2003. Proceedings of 2003 International Symposium on Micromechatronics and Human Science, pp. $303-308$, DOI: 10.1109/MHS.2003.1249959.

[3] J. Everist, W. M. Shen. Mapping opaque and confined environments using proprioception, 2009 ICRA '09. IEEE International Conference on Robotics and Automation, pp. 1041 - 1046, DOI: 10.1109/ROBOT.2009.5152592.

[4] T. Maneewarn, B. Maneechai. Design of Pipe Crawling Gaits for a Snake Robot, IEEE International Conference on Robotics and Biomimetics, 2008. ROBIO 2008, pp. 1 - 6, DOI: 10.1109/ROBIO.2009.4912970.

[5] S. A. Fjerdingen, P. Liljeback, A. A. Transeth. A snake-like robot for internal inspection of complex pipe structures (PIKo), 2009 IEEE/RSJ International Conference on Intelligent Robots and Systems, pp. 5665 - 5671, DOI: 10.1109/IROS.2009.5354751

[6] F. Trebuňa, I. Virgala, M. Kelemen, T. Lipták. Locomotion of Snake Robot through the Pipe. Journal of Automation and Control 2015 (3), No. 3, 135 - 139, DOI: 10.12691/automation-3-3-20.

[7] E. Prada, M. Valášek, I. Virgala, A. Gmiterko, M. Kelemen, M. Hagara, T. Lipták. New approach of fixation possibilities investigation for snake robot in the pipe, 2015 IEEE International Conference on Mechatronics and Automation (ICMA), 2152 - 7431, DOI: 10.1109/ICMA.2015.7237657.

[8] A. Akbarzadeh, Jalil. Safehian, Javad Safehian, H. Kalani. Generating Snake Robot Concertina Locomotion Using a New Dynamic Curve. International Journal of Modeling and Optimization 2011 (1), No. 2, 134 - 140.

[9] R. Silva-Ortigoza, C. Márquez-Sánchez, F. Carrizosa-Corral, V. M. Hernández-Guzmán, J. R. García-Sánchez, H. Taud, M. Marciano-Melchor, J. A. Álvarez-Cedillo. Obstacle Avoidance Task for a Wheeled Mobile Robot - A Matlab-Simulink-Based Didactic Application, MATLAB Applications for the Practical Engineer, 2014, ISBN 978-953-511719-3, DOI: 10.5772/57070.

[10] E. N. Sabudin, R. Omar, Ch. K. Melor. Potential Field Method and their Inherent Approaches for Path Planning. ARPN Journal of Engineering and Applied Sciences 2016 (11), No. 18, $10801-10805$.

[11] C. W. Wampler. Manipulator inverse kinematic solutions based on vector formulations and damped least squares methods. IEEE Transactions on Systems, Man, and Cybernetics 1986 (16), 93-101.

[12] Y. Nakamura, H. Hanafusa. Inverse kinematics solutions with singularity robustness for robot manipulator control. Journal of Dynamic Systems, Measurement, and Control 1986 (108), $163-171$.

[13] F. Duchoň, R. Murár. Modelovanie a riadenie mobilného robota. AT\&P journal Plus2 2006. 
[14] Y. Turygin, P. Božek, Y. Nikitin, E. Sosnovich, A. Abramov. Enhancing the reliability of mobile robots control process via reverse validation. International Journal of Advanced Robotic Systems 2016

[15] J. Úradníček, P. Kraus, M. Musil, M. Bachratý. Investigation of frictional stick-slick effect in disk brake NVH. Journal of Mechanical Engineering - Strojnicky časopis 2017 (67), No. 1, $93-10$.

[16] A. Shala, M. Bruci. Proposed robot scheme with 5 DOF and dynamic modelling using Maple software. Journal of Mechanical Engineering - Strojnícky časopis 2017 (67), No. 2, $101-108$. 